\title{
Effects of diet and exercise interventions on diabetes risk factors in adults without diabetes: meta-analyses of controlled trials
}

\author{
J A D Ranga Niroshan Appuhamy ${ }^{1,2^{*}}$, Ermias Kebreab², Mitchell Simon², Rickey Yada ${ }^{3}$, Larry P Milligan
} and James France ${ }^{1}$

\begin{abstract}
Background and aims: Fasting insulin (FI), fasting glucose (FG), systolic blood pressure (SBP), high density lipoproteins (HDL), triacylglycerides (TAG), and body mass index (BMI) are well-known risk factors for type 2 diabetes. Reliable estimates of lifestyle intervention effects on these factors allow diabetes risk to be predicted accurately. The present meta-analyses were conducted to quantitatively summarize effects of diet and exercise intervention programs on FI, FG, SBP, HDL, TAG and BMI in adults without diabetes.

Materials and methods: MEDLINE and EMBASE were searched to find studies involving diet plus exercise interventions. Studies were required to use adults not diagnosed with type 2 diabetes, involve both dietary and exercise counseling, and include changes in diabetes risk factors as outcome measures. Data from 18, 24, 23, 30, 29 and 29 studies were used for the analyses of FI, FG, SBP, HDL, TAG and BMI, respectively. About $60 \%$ of the studies included exclusively overweight or obese adults. Mean age and BMI of participants at baseline were 48 years and $30.1 \mathrm{~kg} / \mathrm{m}^{2}$. Heterogeneity of intervention effects was first estimated using random-effect models and explained further with mixed-effects models.

Results: Adults receiving diet and exercise education for approximately one year experienced significant $(P<0.001)$ reductions in $\mathrm{Fl}(-2.56 \pm 0.58 \mathrm{mU} / \mathrm{L}), \mathrm{FG}(-0.18 \pm 0.04 \mathrm{mmol} / \mathrm{L})$, SBP $(-2.77 \pm 0.56 \mathrm{~mm} \mathrm{Hg})$, TAG $(-0.258 \pm 0.037 \mathrm{mmol} / \mathrm{L})$ and BMI $\left(-1.61 \pm 0.13 \mathrm{~kg} / \mathrm{m}^{2}\right)$. These risk factor changes were related to a mean calorie intake reduction of $273 \mathrm{kcal} / \mathrm{d}$, a mean total fat intake reduction of $6.3 \%$, and 40 minutes of moderate intensity aerobic exercise four times a week. Lifestyle intervention did not have an impact on HDL. More than $99 \%$ of total variability in the intervention effects was due to heterogeneity. Variability in calorie and fat intake restrictions, exercise type and duration, length of the intervention period, and the presence or absence of glucose, insulin, or lipid abnormalities explained $23-63 \%$ of the heterogeneity.

Conclusions: Calorie and total fat intake restrictions coupled with moderate intensity aerobic exercises significantly improved diabetes risk factors in healthy normoglycemic adults although normoglycemic adults with glucose, insulin, and lipid abnormalities appear to benefit more.
\end{abstract}

\footnotetext{
*Correspondence: ranga.appuhamy@gmail.com

'Department of Animal and Poultry Science, Centre for Nutrition Modelling,

University of Guelph, Guelph N1G 2 W1, Ontario, Canada

${ }^{2}$ Department of Animal Science, University of California, One Shield Avenue,

Davis, CA 95616, USA

Full list of author information is available at the end of the article
} 


\section{Introduction}

High prevalence of type 2 diabetes is strongly associated with obesity and lack of physical activity. Type 2 diabetes is a major cause of kidney failure, lower-limb amputation, blindness, heart disease and is a leading cause of death among adults in Western countries [1]. Consequently, diabetes creates a major financial burden on national healthcare systems representing, for example, more than $10 \%$ of total healthcare expenditures in the USA, Canada and Europe [2].

Type 2 diabetes is a predictable and preventable disease [3]. In addition to pharmacological interventions, type 2 diabetes can be effectively prevented or delayed by lifestyle changes targeting diet and physical activity improvements [4]. Increases in fasting insulin (FI), fasting glucose (FG), systolic blood pressure (SBP), high density lipoproteins (HDL), triacylglycerides (TAG), and body mass index (BMI) are associated with increased risk of developing diabetes and are often used in mathematical models for predicting the risk of developing diabetes [5-7]. Reliable estimates of risk factor responses to lifestyle interventions can improve the accuracy of diabetes risk predictions and assist in planning effective diabetes prevention programs.

Several published studies have investigated the effects of diet and exercise interventions on diabetes risk factors but the estimated effect sizes are inconsistent across studies. Meta-analyses are widely used to compare and combine treatment effects across studies and achieve consensus about the overall treatment effect size. However, conclusions drawn from combining data can be misleading, especially if the individual studies and datasets are considerably different. Therefore, estimation and explanation of the between-study variability or heterogeneity of effect sizes should be an important goal in undertaking meta-analyses. Meta-analyses using random-effect models assume that the studies are a random sample of the entire population of studies, allowing inferences to be generalized beyond the studies included. Random-effect metaanalyses also allow for estimation and exploration of heterogeneity [8].

Meta-analyses have previously been published on lifestyle intervention effects in adults diagnosed with type 2 diabetes $[9,10]$. However, the present study focused on effects among individuals without diabetes as these are directly related to diabetes prevention rather than management. Yamaoka and Tango [11], Gillies et al. [12] and Norris et al. [13] published meta-analyses summarizing the efficacy of diet and exercise interventions among adults with pre-diabetes or impaired glucose tolerance (IGT). We decided to focus on lifestyle intervention effects irrespective of pre-diabetic risk categories. Evidence suggesting the need for lower cutoff levels for diabetes risk categories in Western populations [14] and including more studies in the analyses provided the impetus for this decision. Besides obtaining estimates of effect sizes for overall lifestyle intervention, we were also interested in examining the effects of important intervention aspects such as calorie and fat intake restrictions and improvements in frequency and duration of exercise.

The objectives of the present meta-analyses were to quantitatively summarize 1 ) overall effect size of lifestyle education programs targeting both diet and physical exercise modifications and 2) effects of important dietary and exercise attributes on FI, FG, SBP, HDL, TAG and $\mathrm{BMI}$ in adults without diabetes in Western populations.

\section{Methods}

\section{Literature search}

Studies involving both diet and physical exercise interventions where FI, FG, SBP, TAG, HDL and/or BMI were major outcome measures were searched. Study participants were required to be from Western populations, where the majority is Caucasian. This distinction was made to minimize the potential heterogeneity of intervention effects due to ethnic differences. Study participants in the control group were required to continue with their regular exercise and dietary habits and not receive any diet or exercise counseling prior to or during the study period. MEDLINE and EMBASE computer searches [15] were carried out for articles describing human clinical trials published in English before June 30, 2012 using the keywords: "diet or weight loss", "exercise or physical activity", "diabetes risk or cardiovascular risk", "obese or overweight" and "men and women". Different combinations of key words were searched in both MEDLINE and EMBASE (Table 1). A total of 894 (278 with MEDLINE and 616 with EMBASE) studies were retrieved from the computer searches. Six hand-searched articles were additionally included (Figure 1). 474 duplicates were removed leaving 420 records to be screened. Two authors separately screened the abstracts of the 420 articles and excluded 137 articles because they were about surveys, feasibility studies, trial designs or mathematical and statistical model analyses (Figure 1). Moreover, some of the excluded studies were not from Western countries. The remaining 283 full text articles were assessed for eligibility to be included. The eligibility criteria were 1) trials included adult (men and/or women) participants who were not diagnosed with diabetes, 2) intervention involved both dietary and exercise counseling, 3) dietary counseling targeted calorie and macronutrient intake modifications, 4) outcome measures included changes in diabetes risk factors compared to a control group, 5) availability of mean and variance measures of risk factor changes from baseline in both control and intervention groups, and 6) availability of information on macronutrient intake changes of intervention and control participants. A total of 249 articles were excluded as they did not meet the eligibility criteria 
Table 1 Number of articles retrieved from MEDLINE and EMBASE databases for different key word combinations

\begin{tabular}{lcc}
\hline Keywords & MEDLINE & EMBASE \\
\hline Diet, exercise, obese, diabetes risk, men, women & 27 & 56 \\
$\begin{array}{l}\text { Diet, exercise, overweight, diabetes risk, } \\
\text { men, women }\end{array}$ & 29 & 50 \\
$\begin{array}{l}\text { Diet, exercise, obese, cardiovascular risk, } \\
\text { men, women }\end{array}$ & 32 & 63 \\
$\begin{array}{l}\text { Diet, exercise, overweight, cardiovascular risk, } \\
\text { men, women }\end{array}$ & 34 & 69 \\
$\begin{array}{l}\text { Diet, physical activity, obese, diabetes risk, } \\
\text { men, women }\end{array}$ & 29 & 67 \\
$\begin{array}{l}\text { Diet, physical activity, overweight, diabetes } \\
\text { risk, men, women }\end{array}$ & 28 & 84 \\
$\begin{array}{l}\text { Diet, physical activity, obese, cardiovascular } \\
\text { risk, men, women }\end{array}$ & 33 & 77 \\
$\begin{array}{l}\text { Diet, physical activity, overweight, cardiovascular } \\
\text { risk, men, women }\end{array}$ & 31 & 97 \\
$\begin{array}{l}\text { Weight loss, exercise, obese, diabetes risk, } \\
\text { men, women } \\
\begin{array}{l}\text { Weight loss, exercise, overweight, diabetes risk, } \\
\text { men, women }\end{array}\end{array}$ & 17 & 21 \\
\hline
\end{tabular}

(Figure 1). Two $[16,17]$ of the 34 remaining articles reported risk factors for men and women separately. The male and female groups in these reports were considered two separate studies leaving 36 studies for quality assessment. Quality of the selected studies was evaluated by assessing the risk of four biases [18]: 1) selection bias representing systematic differences between baseline characteristics of intervention and control groups, 2) performance bias regarding exposure to factors other than the interventions of interest, 3) attrition bias involving systematic differences between groups in withdrawals from a study, and 4) publication bias assessed as described below. Baseline characteristics of study participants were not considerably different between control and intervention arms in any of the 36 studies. Although some studies encouraged increased fiber intake and reduced cholesterol intake besides macronutrient intake modifications, these studies were kept in the dataset because fiber and cholesterol intake effects can be accounted for in the analyses as described below. Most of the studies reported no systematic differences between withdrawal groups. However, two articles were excluded because the studies had considerable dropout rates $(>30 \%)$ with no note on the similarities or differences of the withdrawal groups (Figure 1). Although, the statistical significance ( $P$-values) of the intervention effects did not change, the magnitude of the effect sizes changed notably when these two studies were excluded. Since

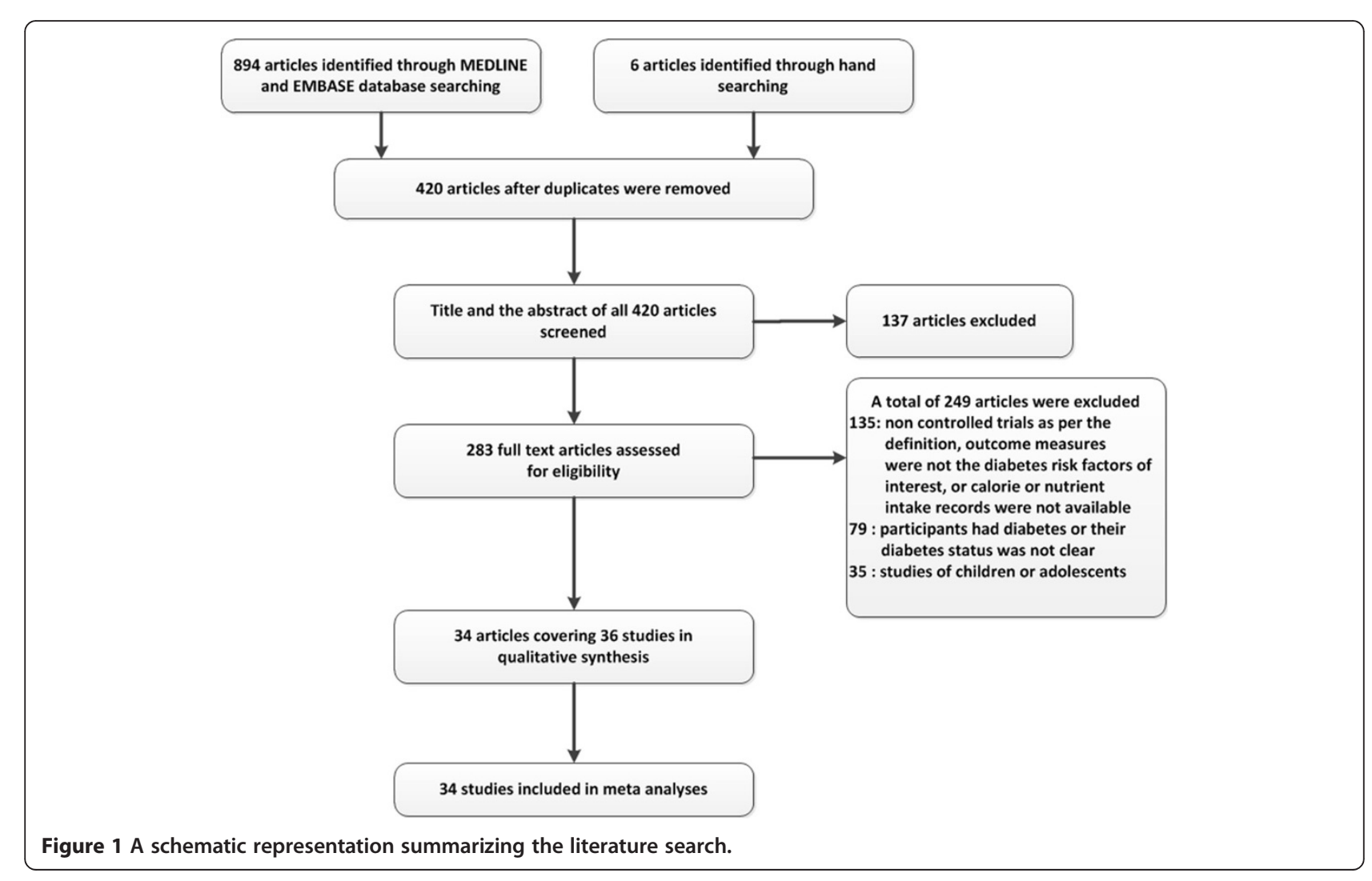


obtaining accurate effect size estimates for lifestyle interventions regarding diabetes risk was the main objective of the present study, we proceeded with the meta-analyses without these two studies. Therefore, the final data set used for the meta-analyses contained 34 studies (Figure 1 and Table 2).

\section{Data and variables}

Data were extracted and put onto structured Excel datasheets designed to capture relevant information in a concise manner. Two authors conducted the screening and data extraction independently using a common set of instructions prepared for the relevant tasks. Sample size (N), mean risk factor levels at baseline and standard deviation (SD) of FI, FG, SBP, HDL, TAG and BMI changes during intervention of the control and intervention arms were essential to the analyses. Mean FI and BMI values were recorded in $\mathrm{mU} / \mathrm{L}$ and $\mathrm{kg} / \mathrm{m}^{2}$, respectively, while the other risk factors were recorded in $\mathrm{mmol} / \mathrm{L}$. In the absence of a reported SD, if 95\% confidence intervals for the risk factor changes were provided, the SD was calculated assuming the changes were normally distributed. In a few cases, the SD was calculated using the $P$-values for risk factor changes from baseline and corresponding test statistics. $\mathrm{N}$ was the number of participants completing each treatment and undergoing post-treatment risk factor measurements. Information on energy $(\mathrm{kcal} / \mathrm{d})$ and the macronutrients: carbohydrate, protein, total fat, and saturated fat intake [\% of energy intake $(\% \mathrm{E})]$ at baseline and at the end of the interventions were recorded in separate columns of the datasheet. When the macronutrient intakes were reported in $\mathrm{g} / \mathrm{d}$ (along with daily energy intake), the $\% \mathrm{E}$ values were calculated using Atwater energy equivalents [19]. Energy and macronutrient intake changes (from baseline) in the control and intervention groups were calculated separately. Difference $(d)$ between these changes mean values are given in Table 3 was then calculated and used in the statistical models.

\section{$d=$ Nutrient intake change in intervention group-Change in control group}

When the nutrient intake changes of control participants were not available, they were assumed to be unchanged from baseline during the interventions. With respect to exercise interventions, two explanatory variables: the number of exercise sessions per week (ES) and minutes of exercise per session (EM) were created. Two binary variables were also created to represent presence or absence of dietary counseling regarding fiber intake increase (FB) and cholesterol intake reduction $(\mathbf{C H})$. As some exercise intervention programs included resistance training sessions, a binary variable (ERT; $1=$ presence or $0=$ absence) was created to examine the impact of resistance training on the diabetes risk factors. When actual ES and EM measures were not available, they were assumed to be equivalent to the exercise intervention guidelines (Table 2). Some studies exclusively recruited people with abnormalities such as impaired glucose tolerance (IGT), insulin resistance (IR), or metabolic syndrome (MetS). Another binary variable (ABN) was created to identify studies that exclusively used participants with such abnormalities. Average age in years, study duration in months and the fraction of women in the study population (ranging from 0 , only men to 1 , only women) were also extracted. When the biomarker levels were measured at multiple time points, the measures of the nutrient intake at the latest time point were used. For some studies, the selected time point was after a follow-up period. Therefore, an additional binary variable (IFU) was generated to represent presence or absence of a follow-up period.

\section{Statistical analyses}

Separate meta-analyses were conducted for each biomarker using the metafor (version 1.6-0) package in $\mathrm{R}$ (version 2.12.2) [50]. Mean difference (MD) was chosen as the effect size measure as it allows effect size interpretation in the original units of risk factor measurement.

\section{$M D=$ Mean riskfactor change (Intervention $)-$ Mean riskfactor change (Control)}

This choice was further supported by the fact that the metafor package allows for weighting individual studies for corresponding sample variance as described below [50]. The forest plots of each risk factor were also constructed using MD (Figures 2, 3, and 4). In addition to $\mathrm{MD}$, forest plots give average sample size, intervention duration, and absolute risk factor changes in the control and intervention arms.

Let:

$$
y_{i}=\theta_{i}+e_{i}
$$

where $y_{i}$ is the observed effect size or MD in the $i^{\text {th }}$ study, $\theta_{i}$ is the corresponding (unknown) true effect size, $e_{i}$ is the sampling error $\left[e_{i} \sim N\left(0, v_{i}\right)\right]$. Sampling variances (i.e., $v_{i}$ ) are assumed to be known and remained fixed during estimation in order to weight the individual studies when estimating model parameters [50]. Betweenstudy variability (heterogeneity) of the true effects $\theta_{i}$ was assumed to be purely random, leading to random-effect models given by:

$$
\theta_{i}=\mu+u_{i}
$$

where $\theta_{i}$ is the true effect size (e.g., MD) in the $i^{\text {th }}$ study, $\mu$ is the overall true effect size, and $u_{i}$ is the random deviation from the overall effect size $\left(u_{i} \sim N\left(0, \tau^{2}\right)\right)$, which was unknown but estimated from the data. The true effects were normally distributed with mean $\mu$ and 
Table 2 Summary description of the diet plus exercise intervention studies included in the analyses

\begin{tabular}{|c|c|c|c|c|c|c|c|c|}
\hline Study & $\begin{array}{c}\text { Authors and } \\
\text { year }\end{array}$ & Country & $\mathrm{Age}^{1}$ & Women $^{2}$ & $\begin{array}{l}\text { IGT/IR/ } \\
\text { MetS }^{3}\end{array}$ & $\mathrm{BMI}^{4}$ & Diet intervention guidelines 5 & $\begin{array}{l}\text { Exercise intervention } \\
\text { guidelines }^{5}\end{array}$ \\
\hline 1 & $\begin{array}{l}\text { Straznicky et al. } \\
2012[20]\end{array}$ & Australia & 55 & 0.41 & MS & 32.7 & $\begin{array}{l}\sim 650 \mathrm{kcal} / \mathrm{d} \text { restriction, } 22 \% \\
\text { protein, 30\% TF, 9\% SF }\end{array}$ & $\begin{array}{l}\text { aerobic (biking), } 40 \mathrm{~min} / \mathrm{S} \text {, } \\
\text { 3-4 S/wk }\end{array}$ \\
\hline 2 & $\begin{array}{l}\text { Blumenthal et al. } \\
2010[21]\end{array}$ & USA & 52 & 0.69 & NA & 33.3 & $\sim 500 \mathrm{kcal} / \mathrm{d}$ restriction, $27 \% \mathrm{TF}$ & $\begin{array}{l}\text { aerobic (biking and walking), } \\
40 \mathrm{~min} / \mathrm{S}, 3-4 \mathrm{~S} / \mathrm{wk}\end{array}$ \\
\hline 3 & $\begin{array}{l}\text { Ibanez et al. } 2010 \\
{[22]}\end{array}$ & Spain & 50 & 1.00 & NA & 35.0 & $500 \mathrm{kcal} / \mathrm{d}$ restriction & $\begin{array}{l}\text { resistance training, } 45-60 \mathrm{~min} / \mathrm{S} \text {, } \\
2 \text { S/wk }\end{array}$ \\
\hline 4 & $\begin{array}{l}\text { Straznicky et al. } \\
2010 \text { [23] }\end{array}$ & Australia & 55 & 0.41 & MS & 32.4 & $\begin{array}{l}\sim 500 \mathrm{kcal} / \mathrm{d} \text { restriction, } 30 \% \mathrm{TF}, \\
9 \% \mathrm{SF}\end{array}$ & aerobic (biking), $40 \mathrm{~min} / \mathrm{S}, 3 \mathrm{~S} / \mathrm{wk}$ \\
\hline 5 & $\begin{array}{l}\text { Roumen et al. } \\
2008 \text { [24] }\end{array}$ & Netherlands & 56 & 0.45 & IGT & 29.4 & $\begin{array}{l}\text { reduced caloric and reduced } \\
\text { fat diet }\end{array}$ & $\begin{array}{l}\text { aerobic plus resistant training, } \\
30 \mathrm{~min} / \mathrm{S}, 5 \mathrm{~S} / \mathrm{wk}\end{array}$ \\
\hline 6 & $\begin{array}{l}\text { Herder et al. } 2009 \\
\text { [25] }\end{array}$ & Finland & 56 & 0.50 & IGT & 31.2 & $<30 \% \mathrm{TF},<10 \% \mathrm{SF}$ & $\begin{array}{l}\text { endurance training, }>30 \mathrm{~min} / \mathrm{S} \text {, } \\
\sim 6 \mathrm{~S} / \mathrm{wk}\end{array}$ \\
\hline 7 & $\begin{array}{l}\text { Mosca et al. } 2008 \\
{[26]}\end{array}$ & USA & 48 & 0.66 & NA & 28.1 & low-SF and low-cholesterol diet & $\begin{array}{l}\text { moderate physical activity (brisk } \\
\text { walking), 30-60 min/s }\end{array}$ \\
\hline 8 & $\begin{array}{l}\text { Morgan et al. } \\
2009[27]\end{array}$ & UK & 41 & 0.74 & NA & 31.6 & $\begin{array}{l}\text { Rosemary Conley's controlled- } \\
\text { calorie low-fat diet }\end{array}$ & $\begin{array}{l}\text { Rosemary Conley's Fitness plan } \\
\text { with weekly classes }\end{array}$ \\
\hline 9 & $\begin{array}{l}\text { Dale et al. } 2009 \\
\text { [28] }\end{array}$ & New Zealand & 46 & 0.67 & $\mathbb{R}$ & 34.6 & $\begin{array}{l}400 \mathrm{kcal} / \mathrm{d} \text { restriction, } 27 \% \mathrm{TF} \text {, } \\
9 \% \mathrm{SF}\end{array}$ & $\begin{array}{l}\text { high intensity training, } 30 \mathrm{~min} / \mathrm{S} \text {, } \\
5 \mathrm{~S} / \mathrm{wk}\end{array}$ \\
\hline 10 & $\begin{array}{l}\text { Meckling and } \\
\text { Sherfey, } 2007 \text { [29] }\end{array}$ & Canada & 43 & 1.00 & NA & 29.9 & $\begin{array}{l}500 \mathrm{kcal} / \mathrm{d} \text { restriction, high } \\
\text { protein }(37 \%) \text { diet }\end{array}$ & $\begin{array}{l}\text { endurance training, } 36 \mathrm{~min} / \mathrm{S} \text {, } \\
3 \mathrm{~S} / \mathrm{wk}\end{array}$ \\
\hline 11 & $\begin{array}{l}\text { Burke et al. } 2007 \\
\text { [30] }\end{array}$ & Australia & 56 & 0.56 & NA & 30.1 & $<30 \%$ TF,$<10 \%$ SF & $\begin{array}{l}\text { moderate intensity, } 30 \mathrm{~min} / \mathrm{S} \text {, } \\
\text { most days/wk }\end{array}$ \\
\hline 12 & Bo et al. 2007 [31] & Italy & 56 & 0.58 & MS & 30.0 & reduced TF and SF intake & $\begin{array}{l}\text { moderate intensity (i. e. brisk } \\
\text { walking), } 150 \mathrm{~min} / \mathrm{wk}\end{array}$ \\
\hline 13 & $\begin{array}{l}\text { Arciero et al. } 2006 \\
\text { [32] }\end{array}$ & USA & 43 & 0.48 & NA & 27.8 & $\begin{array}{l}\text { high protein (40\%) and low fat } \\
\text { (20\%) diet }\end{array}$ & $\begin{array}{l}\text { resistance and cardiovascular } \\
\text { training, } 20 \mathrm{~min} / \mathrm{S}, 4-6 \mathrm{~S} / \mathrm{wk}\end{array}$ \\
\hline 14 & $\begin{array}{l}\text { Brekke et al. } 2005 \\
\text { [33] }\end{array}$ & Sweden & 42 & 0.37 & NA & 26.1 & $<30 \%$ TF intake, $<10 \%$ SF intake & $\begin{array}{l}\text { walking or more intensive } \\
\text { exercise, } 30 \mathrm{~min} / \mathrm{S}, 4-5 \mathrm{~S} / \mathrm{wk}\end{array}$ \\
\hline 15 & $\begin{array}{l}\text { Watkins et al. } 2003 \\
\text { [34] }\end{array}$ & USA & 50 & 0.50 & NA & 33.7 & $500 \mathrm{kcal} / \mathrm{d}$ restriction, $<20 \% \mathrm{TF}$ & $\begin{array}{l}\text { cycle ergometry and jogging, or } \\
\text { walking, } \sim 60 \mathrm{~min} / \mathrm{S}, 3-4 \mathrm{~S} / \mathrm{wk}\end{array}$ \\
\hline 16 & $\begin{array}{l}\text { Lindstrom et al. } \\
2003[35]\end{array}$ & Finland & 55 & 0.66 & IGT & 31.3 & $\begin{array}{l}200 \mathrm{kcal} / \mathrm{d} \text { restriction, }<30 \% \mathrm{TF} \\
<10 \% \mathrm{SF}\end{array}$ & $\begin{array}{l}\text { endurance exercise \& resistance } \\
\text { training, }>30 \mathrm{~min} / \mathrm{S}\end{array}$ \\
\hline 17 & $\begin{array}{l}\text { Esposito et al. } \\
2003 \text { [36] }\end{array}$ & Italy & 35 & 1.00 & NA & 34.5 & $\begin{array}{l}1400 \mathrm{kcal} / \mathrm{d}, 55 \% \text { carbohydrate, } \\
30 \% \mathrm{TF},<10 \% \mathrm{SF}\end{array}$ & $\begin{array}{l}\text { aerobic exercise (walking and } \\
\text { swimming) }\end{array}$ \\
\hline 18 & $\begin{array}{l}\text { Mensink et al. } \\
2003[37]\end{array}$ & Netherlands & 56 & 0.43 & IGT & 29.5 & $\begin{array}{l}>55 \% \text { carbohydrate, }<30 \% \mathrm{TF} \\
<10 \% \mathrm{SF}\end{array}$ & $\begin{array}{l}\text { moderate physical activity, } \\
>30 \mathrm{~min} / \mathrm{S}, 5 \mathrm{~S} / \mathrm{wk}\end{array}$ \\
\hline 19 & $\begin{array}{l}\text { McAuley et al. } \\
2002[38]\end{array}$ & New Zealand & 46 & 0.71 & $\mathbb{R}$ & 34.5 & $\begin{array}{l}400 \mathrm{kcal} / \mathrm{d} \text { restriction, } 27 \% \mathrm{TF} \text {, } \\
9 \% \mathrm{SF}\end{array}$ & $\begin{array}{l}\text { Moderate exercise plus resistance } \\
\text { training, }>20 \mathrm{~min} / \mathrm{S}, 5 \mathrm{~S} / \mathrm{wk}\end{array}$ \\
\hline 20 & $\begin{array}{l}\text { Miller et al. } 2002 \\
\text { [39] }\end{array}$ & USA & 54 & 0.62 & NA & 33.7 & $\begin{array}{l}500 \mathrm{kcal} / \mathrm{d} \text { restriction, } 27 \% \mathrm{TF} \text {, } \\
6 \% \mathrm{SF}\end{array}$ & $\begin{array}{l}\text { aerobic (brisk walking and } \\
\text { biking), 30-45 } \mathrm{min} / \mathrm{S}, 3 \mathrm{~S} / \mathrm{wk}\end{array}$ \\
\hline 21 & $\begin{array}{l}\text { Reseland et al. } \\
2001[40]\end{array}$ & Norway & 45 & 0.00 & MS & 27.5 & $400 \mathrm{kcal} / \mathrm{d}$ restriction, $<30 \% \mathrm{TF}$ & endurance exercise, $1 \mathrm{~h} / \mathrm{S}, 3 \mathrm{~S} / \mathrm{wk}$ \\
\hline 22 & $\begin{array}{l}\text { Oldroyd et al. } \\
2001 \text { [41] }\end{array}$ & UK & 58 & 0.40 & IGT & 30.2 & $\begin{array}{l}<30 \% \text { TF intake, } \sim 50 \% \\
\text { carbohydrate }\end{array}$ & $\begin{array}{l}\text { aerobic exercise, } 20-30 \mathrm{~min} / \mathrm{S} \\
\text { 2-3 S/wk }\end{array}$ \\
\hline 23 & $\begin{array}{l}\text { Kuller et al. } 2001 \\
\text { [42] }\end{array}$ & USA & 47 & 1.00 & NA & 25.0 & $\begin{array}{l}\text { Calorie restriction upto } 1300 \mathrm{kcal} \text {, } \\
25 \% \mathrm{TF}, 7 \% \mathrm{SF}\end{array}$ & $\begin{array}{l}\text { increasing physical activity to } \\
1250 \text { kcal expended weekly }\end{array}$ \\
\hline 24 & $\begin{array}{l}\text { Ornish et al. } 1998 \\
\text { [43] }\end{array}$ & USA & 60 & 0.09 & NA & 26.9 & $10 \%$-fat vegetarian diet & $\begin{array}{l}\text { moderate-intensity aerobic, } 1 \mathrm{~h} / \mathrm{S} \text {, } \\
5 \mathrm{~S} / \mathrm{wk}\end{array}$ \\
\hline 25 & $\begin{array}{l}\text { Stefanick et al. } \\
1998 \text { (female) [16] }\end{array}$ & USA & 57 & 1.00 & NA & 25.6 & $<30 \%$ TF intake, $<7 \%$ SF intake & $\begin{array}{l}\text { aerobic (jogging and brisk } \\
\text { walking), } 60 \mathrm{~min} / \mathrm{S}, 3 \mathrm{~S} / \mathrm{wk}\end{array}$ \\
\hline 26 & $\begin{array}{l}\text { Stefanick et al. } \\
1998 \text { (male) [16] }\end{array}$ & USA & 48 & 1.00 & NA & 27.8 & $<30 \%$ TF intake, $<7 \%$ SF intake & $\begin{array}{l}\text { aerobic (jogging and brisk } \\
\text { walking), } 60 \mathrm{~min} / \mathrm{S}, 3 \mathrm{~S} / \mathrm{wk}\end{array}$ \\
\hline
\end{tabular}


Table 2 Summary description of the diet plus exercise intervention studies included in the analyses (Continued)

\begin{tabular}{|c|c|c|c|c|c|c|c|c|}
\hline 27 & $\begin{array}{l}\text { Wing et al. } 1998 \\
\text { [44] }\end{array}$ & USA & 46 & 0.78 & NA & 36.0 & $\begin{array}{l}600-700 \mathrm{kcal} / \mathrm{d} \text { restriction, } 20 \% \mathrm{TF} \\
\text { intake }\end{array}$ & $\begin{array}{l}\text { aerobic (brisk walking), } 60 \mathrm{~min} / \mathrm{S} \text {, } \\
5 \mathrm{~S} / \mathrm{wk}\end{array}$ \\
\hline 28 & $\begin{array}{l}\text { Simkin-Silverman } \\
\text { et al. } 1995 \text { [45] }\end{array}$ & UK & 47 & 1.00 & NA & 25.1 & $<25 \%$ TF intake, $<7 \%$ SF intake & $\begin{array}{l}\text { brisk walking spending } \\
1000 \mathrm{kcal} / \mathrm{wk}, 3-5 \mathrm{~S} / \mathrm{wk}\end{array}$ \\
\hline 29 & $\begin{array}{l}\text { Hellenius et al. } \\
1993[46]\end{array}$ & Sweden & 46 & 0.00 & NA & 25.6 & $\begin{array}{l}12 \% \text { daily calorie and 10\% TF } \\
\text { restriction }\end{array}$ & $\begin{array}{l}\text { aerobic exercise, } 30-45 \mathrm{~min} / \mathrm{S}, \\
2-3 \mathrm{~S} / \mathrm{wk}\end{array}$ \\
\hline 30 & $\begin{array}{l}\text { Svendsen et al. } \\
1993[47]\end{array}$ & Denmark & 54 & 1.00 & NA & 29.7 & $\begin{array}{l}800 \mathrm{kcal} / \mathrm{d} \text { restriction, low fat } \\
(25 \%) \text { diet }\end{array}$ & $\begin{array}{l}\text { aerobic plus resistant training, } \\
75 \mathrm{~min} / \mathrm{S}, 3 \mathrm{~S} / \mathrm{wk}\end{array}$ \\
\hline 31 & $\begin{array}{l}\text { Page et al. } 1993 \\
\text { [48] }\end{array}$ & UK & 40 & 0.23 & IGT & 25.9 & $\begin{array}{l}50-55 \% \text { carbohydrate, 30\% TF, } \\
\text { high fiber }\end{array}$ & $\begin{array}{l}\text { aerobic weight and circuit } \\
\text { training, swimming, }>3 \mathrm{~S} / \mathrm{wk}\end{array}$ \\
\hline 32 & $\begin{array}{l}\text { Schuler et al. } 1992 \\
\text { [49] }\end{array}$ & Germany & 54 & 0.00 & NA & 26.6 & $\begin{array}{l}<20 \% \text { TF, } 65 \% \text { carbohydrate, } \\
\text { PUSF: SF ratio }>1.0\end{array}$ & $\begin{array}{l}\text { daily exercise ( } 75 \% \mathrm{MHR}) \\
>30 \mathrm{~min} / \mathrm{S}\end{array}$ \\
\hline 33 & $\begin{array}{l}\text { Wood et al. } 1991 \\
\text { (male) [17] }\end{array}$ & USA & 38 & 0.00 & NA & 33.5 & $\begin{array}{l}55 \% \text { carbohydrate, } 30 \% \mathrm{TF} \\
<10 \% \mathrm{SF}\end{array}$ & $\begin{array}{l}\text { aerobic (brisk walking and } \\
\text { jogging), } 35 \mathrm{~min} / \mathrm{S}, 3 \mathrm{~S} / \mathrm{wk}\end{array}$ \\
\hline 34 & $\begin{array}{l}\text { Wood et al. } 1991 \\
\text { (female) [17] }\end{array}$ & USA & 38 & 1.00 & NA & 26.3 & $\begin{array}{l}55 \% \text { carbohydrate, } 30 \% \text { TF, } \\
<10 \% \text { SF }\end{array}$ & $\begin{array}{l}\text { aerobic (brisk walking and } \\
\text { jogging), } 35 \mathrm{~min} / \mathrm{S}, 3 \mathrm{~S} / \mathrm{wk}\end{array}$ \\
\hline
\end{tabular}

${ }^{1}$ Average age of participants at baseline (years), ${ }^{2}$ fraction of women participants, ${ }^{3}$ whether participants had abnormalities such as impaired glucose tolerance (IGT), insulin resistance (IR) and metabolic syndrome (MetS) or no abnormalities (NA), ${ }^{4}$ average BMI of participants at baseline $\left(\mathrm{kg} / \mathrm{m}^{2}\right),{ }^{5} \mathrm{TF}=\mathrm{Total}$ fat $(\%$ of energy), $\mathrm{SF}=$ Saturated fat (\% of energy), and $S=$ exercise session.

variance $\tau^{2}$. If $\tau^{2}=0$, homogeneity is implied among true effects across individual studies such that $\mu=\theta$. Heterogeneity $\left(\tau^{2}\right)$ was expressed as a percentage of total variability $\left(\tau^{2}\right.$ plus sample variance) yielding $I^{2}$ statistics.

An $I^{2}$ value greater than $50 \%$ indicates considerable heterogeneity. Thus, for response variables (e.g., intervention effects on diabetes risk factors in the present study) with $I^{2}>50 \%$, the random-effect models were extended to mixed-effect models including fixed effects of explanatory variables (meta-regression analyses) with the potential to explain the heterogeneity in intervention effects. The mixed-effect models were given by:

$$
\theta_{i}=\beta_{0}+\beta_{1} x_{i 1}+\ldots+\beta_{p} x_{i p}+u_{i}
$$

where $\beta_{0}$ is the overall true effect size, $x_{i j}$ is the the value of the $j^{\text {th }}$ explanatory variable $(j=1,2, \ldots, p)$ for the $i^{\text {th }}$ study, $\beta_{j}$ is the change in the true effect size for a unit increase in the $j^{\text {th }}$ explanatory variable and $u_{i} \sim N\left(0, \tau^{2}\right)$. Here, $\tau^{2}$ denotes the amount of residual heterogeneity [50]. The parameters in the mixed-effect models $\left(\beta_{0}, \ldots, \beta_{p}\right)$ were estimated via weighted least squares with weights equal to $1 /\left(v_{i}+\tau^{2}\right)$.

The candidate explanatory variables for the mixed-effect models were energy and macronutrient intake changes, the exercise attributes: ES, EM and ERT, baseline risk factor level, baseline age and BMI, time duration from baseline to the post-intervention risk factor measurements, FB, $\mathrm{CH}, \mathrm{ABN}$, IFU and fraction of women in the study population. Each continuous explanatory variable was centered on its mean before being used in the mixed-effect models. This allows for interpreting the meta-regression coefficients in terms of changes in intervention effect size for a unit change in a continuous explanatory variable from its mean. Mixed-effect models including individual explanatory variables were first fitted to the data. Full multivariate mixed-effect models were then formed including all explanatory variables having notable effects $(P<0.10)$ when fitted individually. Reduced models were formed via stepwise elimination of one explanatory variable at a time. The final mixed-effect models were chosen by comparing reduced models vs. full models using log-likelihood ratio tests with the maximum likelihood method.

\section{Publication bias and influence diagnosis}

Publication bias and presence of influential cases can affect the validity and robustness of the conclusions from a meta-analysis [51,52]. Studies for which the effect size estimates and their variability are extremely sensitive were recognized using a leave-one-out approach. The leave1out function in metafor package was used to execute this influence diagnostic analysis, which is equivalent to a sensitivity analysis. Cook's distance and $\tau^{2}$ estimates $\left(\tau_{\mathrm{D}}^{2}\right)$ were obtained when each study was excluded from the data set. [50]. If a study was related to a Cook's distance $>2$ and $\tau_{\mathrm{D}}^{2}$ two SD below the mean $\tau_{\mathrm{D}}^{2}$ of the other studies, that study was removed from the dataset. Initially, 19, 25, 25, 31, 30, and 30 of the 34 studies were chosen for FI, FG, SBP, HDL, TAG and BMI analyses respectively, but after the influence analyses 18, 24, 23, 30, 29, and 29 studies were retained, respectively. Presence of publication bias was assessed using funnel plots. Asymmetric funnel plots indicate the presence of publication bias. Egger's regression test was used to examine funnel plot asymmetry [50]. None of the funnel plots was found to be significantly asymmetric $(P$-values for funnel plot asymmetry $>0.05)$ indicating absence of publication bias in all cases. 


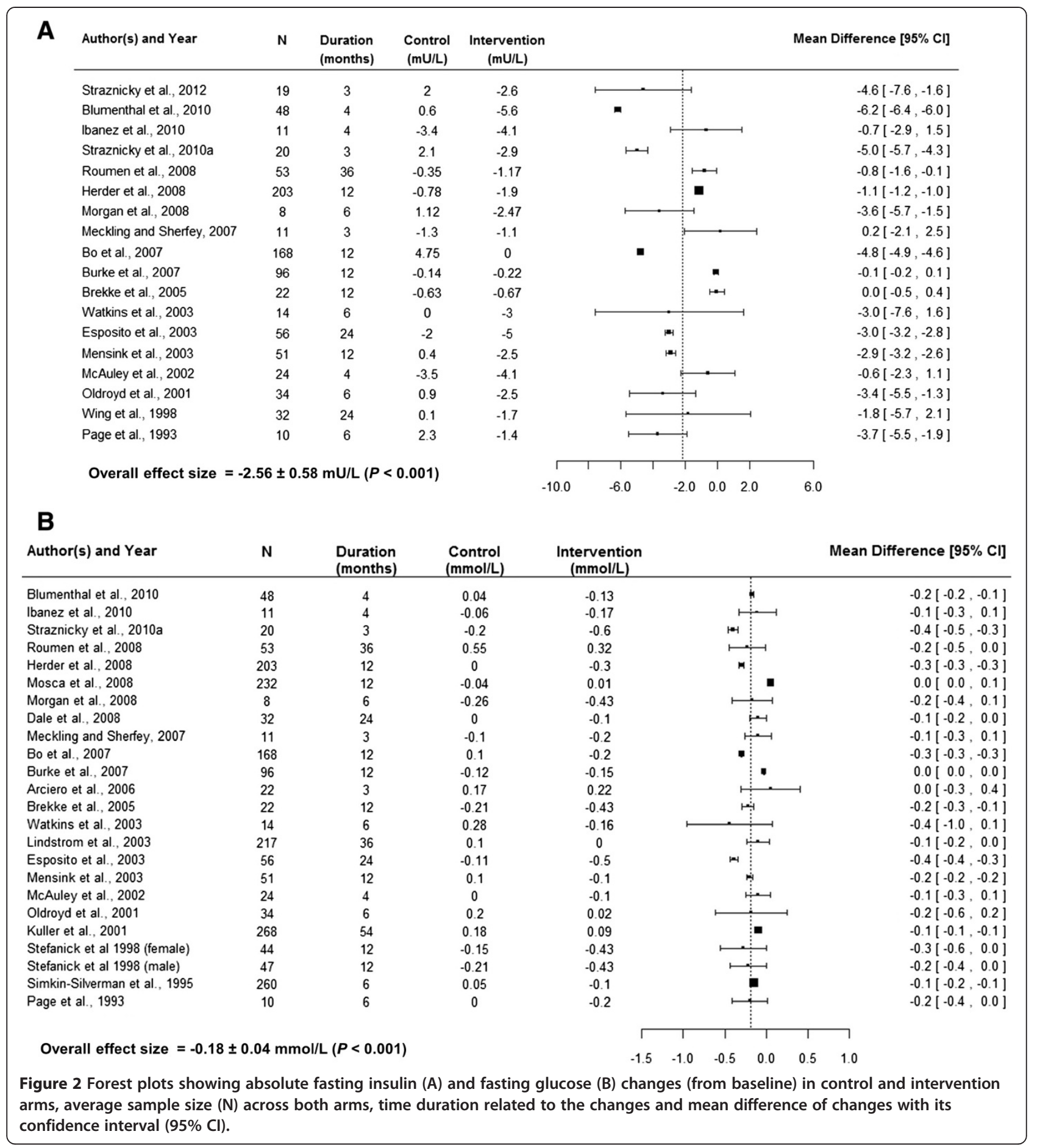

\section{Results}

\section{Study characteristics}

Of the 34 studies included in meta-analyses, 16, 13, and 5 studies were from Europe, North America, and Australia and New Zealand, respectively (Table 2). Information about ethnic composition of participants was not available in all the articles. Based on the articles reporting the ethnic composition, $>60 \%$ of the study participants were
Caucasian. Average age at baseline ranged from 35 to 60 years with a mean of 49 years, indicating that the majority of study participants were middle-aged. Twenty of the 34 studies recruited exclusively overweight and obese individuals. Average baseline BMI varied from 25.0 to $36.0 \mathrm{~kg} / \mathrm{m}^{2}$ with a mean of $30.2 \mathrm{~kg} / \mathrm{m}^{2}$ (Table 3). Twelve of the 34 studies examined diet plus exercise intervention specifically in adults with abnormalities such as IGT, IR, 


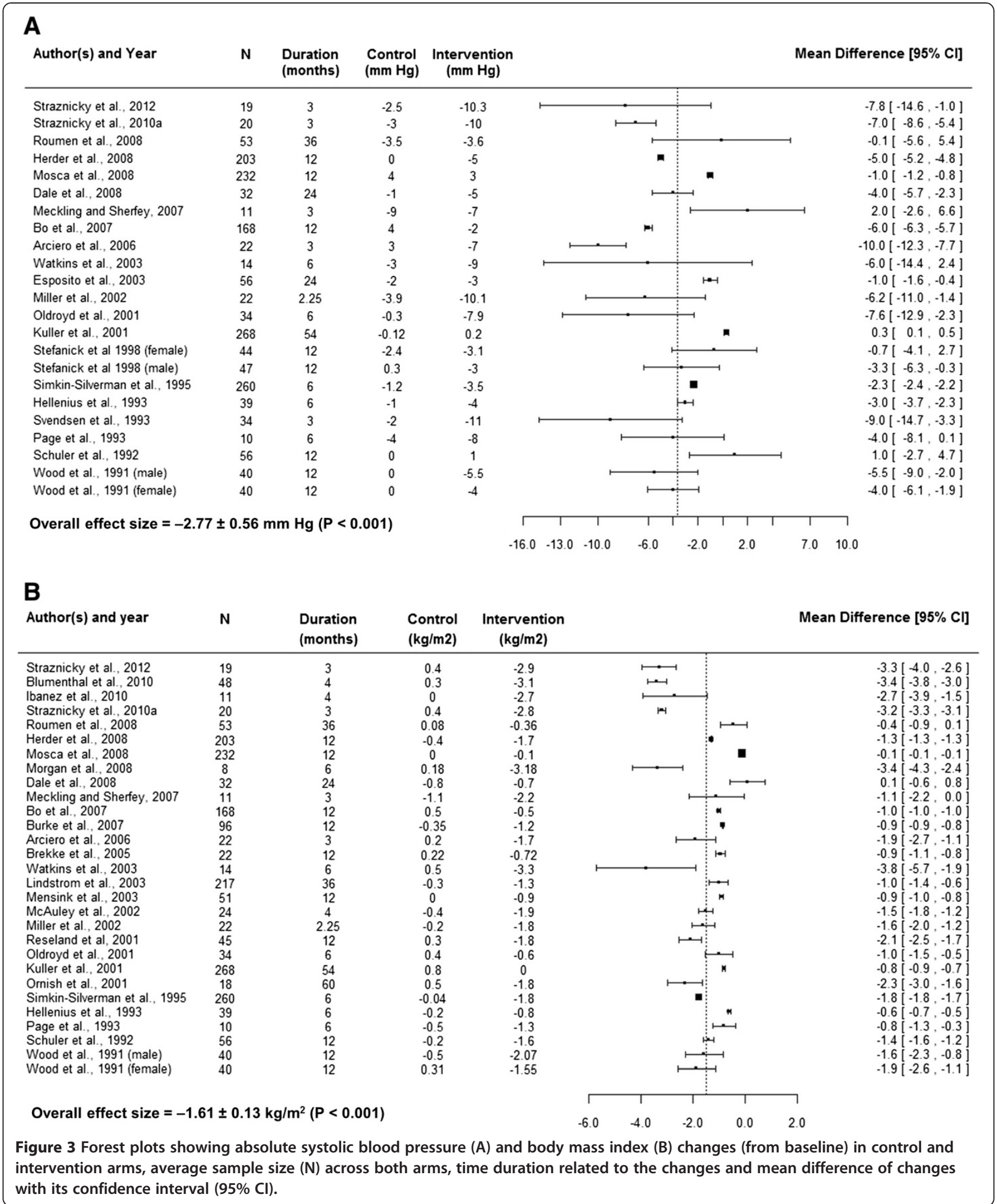

or MetS. Average FG of participants in the individual studies ranged between 4.83 and $6.20 \mathrm{mmol} / \mathrm{L}$, with a mean of $5.55 \mathrm{mmol} / \mathrm{L}$. The respective FI range was 3.0 and $24.1 \mathrm{mU} / \mathrm{L}$ with a mean of $14.9 \mathrm{mU} / \mathrm{L}$. Time duration from baseline risk factor measurements to the postintervention measurements varied from 2 to 60 months with a mean of 14 months (Table 3 ). In a few studies, this duration included a follow-up period in addition to 


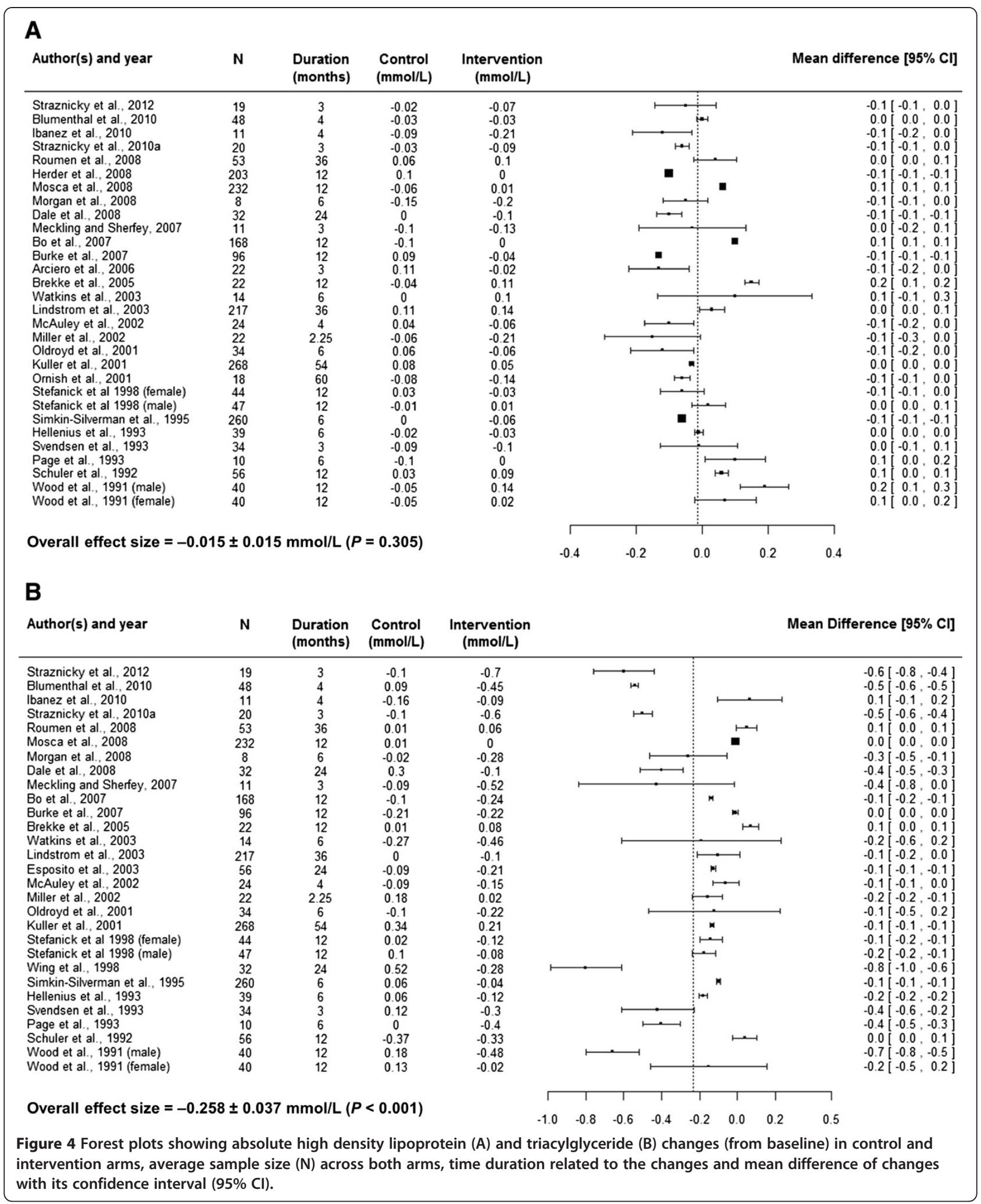

the intervention counselling period. Nine of the studies included only female participants and four used only males while the rest (21 studies) included both males and females. The majority of lifestyle intervention programs aimed to restrict energy intake by $500 \mathrm{kcal} / \mathrm{d}$, total fat $\leq 30 \% \mathrm{E}$, and saturated fat $\leq 10 \% \mathrm{E}$ (Table 2) and 
Table 3 Summary statistics for variables across the 34 studies included in the meta-analyses

\begin{tabular}{|c|c|c|c|}
\hline Variable & Mean $\pm S D^{1}$ & Minimum & Maximum \\
\hline \multicolumn{4}{|l|}{$\begin{array}{l}\text { Energy and macronutrient intake } \\
\text { at baseline }\end{array}$} \\
\hline Calorie intake, kcal/d & $2100 \pm 260$ & 1583 & 2900 \\
\hline Carbohydrate, \%E & $46.6 \pm 5.0$ & 36.5 & 58.5 \\
\hline Protein, \%E & $17.2 \pm 2.6$ & 12.3 & 25 \\
\hline Fat, \%E & $34.7 \pm 3.3$ & 27.5 & 43.5 \\
\hline Saturated fat, \%E & $12.9 \pm 1.8$ & 9 & 16.6 \\
\hline \multicolumn{4}{|l|}{$\begin{array}{l}\text { Energy and macronutrient intake } \\
\text { changes of intervention participants }{ }^{2}\end{array}$} \\
\hline Calorie intake, kcal/d & $-273 \pm 169$ & -828 & 49.5 \\
\hline Carbohydrate, \%E & $2.9 \pm 5.7$ & -14 & 11 \\
\hline Protein, \%E & $3.3 \pm 5.6$ & -3.8 & 21.3 \\
\hline Fat, \%E & $-6.3 \pm 3.8$ & -16.5 & 0.4 \\
\hline Saturated fat, \%E & $-2.9 \pm 2.0$ & -8 & 0.0 \\
\hline \multicolumn{4}{|l|}{ Exercise intervention } \\
\hline Number of sessions per week & $4 \pm 1.0$ & 2 & 7 \\
\hline Session length (minutes) & $41 \pm 13$ & 20 & 75 \\
\hline \multicolumn{4}{|l|}{$\begin{array}{l}\text { Risk factor level measurements } \\
\text { at baseline }\end{array}$} \\
\hline $\mathrm{Fl}$ & $14.9 \pm 5.4$ & 3.0 & 24.1 \\
\hline FG & $5.55 \pm 0.4$ & 4.83 & 6.20 \\
\hline SBP & $128 \pm 10$ & 110 & 143 \\
\hline $\mathrm{HDL}$ & $1.25 \pm 0.2$ & 0.89 & 1.74 \\
\hline TAG & $1.76 \pm 0.9$ & 0.85 & 5.84 \\
\hline BMl & $30.2 \pm 3.3$ & 25.0 & 36.0 \\
\hline Age at baseline, years & $49 \pm 7$ & 35 & 60 \\
\hline Fraction of women participants & $0.59 \pm 0.3$ & 0 & 1 \\
\hline $\begin{array}{l}\text { Duration of intervention } \\
\text { (months) }^{3}\end{array}$ & $14 \pm 3.5$ & 2 & 60 \\
\hline
\end{tabular}

${ }^{1} S D$, Standard deviation.

${ }^{2}$ Change from baseline compared to that of control participants.

${ }^{3}$ Duration from baseline to the risk factor level measurements used in the analyses.

to improve physical activity through moderate-intensity aerobic exercises such as brisk walking, jogging, cycling, and swimming. About one-third of the intervention programs included instructions for resistance training. Overall, the intervention programs involved an average of 4 exercise sessions a week each lasting 41 minutes (Table 3).

\section{Heterogeneity}

Random-effect model analyses (Table 4) revealed substantial heterogeneity $\left(\tau^{2}, P<0.001\right)$ of lifestyle intervention effects on all risk factors. The $I^{2}$ estimates $\left(\tau^{2} / \tau^{2}+\right.$ sample variance) were $>99.0 \%$ in all cases (Table 4 ) indicating that between-study variability of intervention effects $\left(\tau^{2}\right)$ were $>110$ times greater than within-study variability (sample variance). Therefore the random-effect models were extended to mixed-effect models to explore heterogeneity.

Intervention effect size estimates from mixed-effect models Calorie intake restrictions, abnormalities such as IGT or IR, and resistance training exercises influenced FI responses to lifestyle interventions $(P<0.10)$ and accounted for $60 \%$ of the heterogeneity $\left[\tau^{2}=3.762\right.$ (Table 4 ) vs. 1.523 (Table 5)]. Lifestyle interventions reduced FI by $2.56 \pm$ $0.58 \mathrm{mU} / \mathrm{L}$ (Table 5) in normal adults engaging in moderate intensity aerobic exercise. This decrease in FI was related to a mean $273 \mathrm{kcal} / \mathrm{d}$ restriction (Table 3). The negative parameter estimate of calorie restriction in Table 5 shows that an additional $100 \mathrm{kcal} / \mathrm{d}$ restriction would have reduced FI by an additional $0.68 \pm 0.26 \mathrm{mU} /$ L. On the other hand, incorporation of resistance training into the exercise intervention impeded the expected FI decline by $1.72 \pm 0.81 \mathrm{mU} / \mathrm{L}$. Hence, the expected FI change among adults engaging in resistance training was $-0.84 \mathrm{mU} / \mathrm{L}(-2.56+1.72 \mathrm{mU} / \mathrm{L})$. Regardless of the degree of calorie restriction and exercise intensity, adults with metabolic abnormalities tended $(P=0.082)$ to experience an extra FI reduction of $1.20 \pm 0.69 \mathrm{~mm} \mathrm{Hg}$ (Table 5).

Diet plus exercise interventions reduced FG by $0.18 \pm$ $0.04 \mathrm{mmol} / \mathrm{L}(P<0.001)$ in normoglycemic healthy adults. The metabolic abnormality and follow-up period variables considerably explained the heterogeneity of the intervention effects on FG. Heterogeneity was reduced by $23 \%$ when these factors were included in the final model $\left(\tau^{2}=0.013\right.$ vs. 0.010$)$. Participants experienced less (by $0.13 \pm 0.06 \mathrm{mmol} / \mathrm{L}$ ) FG decline as they entered a follow-up period, during which rigorous intervention counselling was not provided. Regardless of undergoing a follow-up period or not, adults with metabolic abnormalities tended $(P=0.098)$ to experience a greater FG decline (by $0.08 \pm 0.05 \mathrm{mmol} / \mathrm{L}$ ) compared to normal adults (Table 5). Diet and exercise interventions reduced SBP by $2.77 \pm 0.56 \mathrm{~mm} \mathrm{Hg}(P<0.001$, Table 5$)$. However, study participants with abnormalities experienced an extra SBP decline of $3.23 \pm 1.00 \mathrm{~mm} \mathrm{Hg}$ (Table 5). Extending intervention by a month reduced the expected SBP decline by $0.13 \pm 0.03 \mathrm{~mm} \mathrm{Hg}$. Calorie restriction increased by $100 \mathrm{kcal} / \mathrm{d}$ was associated with an additional SBP decline of $-0.64 \pm 0.23 \mathrm{~mm} \mathrm{Hg}(P=0.006)$. Presence or absence of abnormalities, intervention duration, and degree of calorie intake restriction explained collectively $63 \%\left[\tau^{2}=7.474\right.$ (Table 4) vs. 2.796 (Table 5) $]$ of heterogeneity of the intervention effect on SBP.

The diet plus exercise interventions did not have a significant impact $(P=0.305)$ on HDL (Table 5). Nonetheless, age of participants at baseline and percentage of women in the study population explained $29 \%$ of heterogeneity in 
Table 4 Heterogeneity $\left(\tau^{2}\right)$ of lifestyle intervention effects and statistical significance of funnel plot asymmetry from random-effect models

\begin{tabular}{|c|c|c|c|c|c|c|}
\hline \multirow[t]{2}{*}{ Risk factor $^{1}$} & \multirow{2}{*}{$\begin{array}{c}\text { Number of } \\
\text { studies included }\end{array}$} & \multirow[t]{2}{*}{$\mathrm{N}^{2}$} & \multicolumn{3}{|c|}{ Heterogeneity ${ }^{2}$} & \multirow{2}{*}{$\begin{array}{c}\text { Funnel plot } \\
{\text { asymmetry }(P \text {-value })^{3}}^{3}\end{array}$} \\
\hline & & & $\overline{P^{2}(\%)^{2}}$ & $\tau^{2}$ & $P$-value & \\
\hline $\mathrm{FI}(\mathrm{mU} / \mathrm{L})$ & 18 & 1756 & 99.5 & $3.762 \pm 1.511$ & $<0.001$ & 0.519 \\
\hline $\mathrm{FG}(\mathrm{mmol} / \mathrm{L})$ & 24 & 3897 & 99.4 & $0.013 \pm 0.005$ & $<0.001$ & 0.774 \\
\hline $\mathrm{SBP}(\mathrm{mm} \mathrm{Hg})$ & 23 & 3443 & 99.4 & $7.474 \pm 2.894$ & $<0.001$ & 0.771 \\
\hline $\mathrm{HDL}(\mathrm{mmol} / \mathrm{L})$ & 30 & 4218 & 99.8 & $0.007 \pm 0.002$ & $<0.001$ & 0.577 \\
\hline TAG (mmol/L) & 29 & 3908 & 99.8 & $0.050 \pm 0.015$ & $<0.001$ & 0.855 \\
\hline BMI $\left(\mathrm{kg} / \mathrm{m}^{2}\right)$ & 29 & 4160 & 99.8 & $0.881 \pm 0.256$ & $<0.001$ & 0.587 \\
\hline
\end{tabular}

${ }^{1}$ FI: Fasting insulin, FG: Fasting glucose, SBP: Systolic blood pressure, HDL: High density lipoprotein, TAG: Triacylglyceride, BMI: Body mass index.

${ }^{2}$ total number of participants, who concluded the interventions.

${ }^{2} T^{2}=$ heterogeneity $\left(\tau^{2}\right)$ expressed as a percentage of total variance $\left(\tau^{2}+\right.$ sample error).

${ }^{3}$ From Egger's regression test.

HDL changes in intervention participants compared to control participants. The lifestyle interventions reduced TAG by $0.258 \pm 0.037 \mathrm{mmol} / \mathrm{L}(P<0.001)$. This drop in TAG was related to a mean net energy intake restriction of $273 \mathrm{kcal} / \mathrm{d}$ and a mean baseline TAG of $1.76 \mathrm{mmol} / \mathrm{L}$ (Table 3). An additional $100 \mathrm{kcal} / \mathrm{d}$ energy restriction would have further improved the decrease in TAG by $0.061 \pm 0.017 \mathrm{mmol} / \mathrm{L}$. A unit increase in TAG at baseline was associated with an additional TAG drop of $0.243 \pm$ $0.076 \mathrm{mmol} / \mathrm{L}$.

Adult participants experienced a $1.61 \pm 0.13 \mathrm{~kg} / \mathrm{m}^{2}$ $(P<0.001)$ BMI decline when engaged in a diet plus exercise lifestyle intervention program. The extent of calorie and total fat intake restrictions, duration of exercise sessions, and baseline BMI considerably affected the degree of BMI change $(P<0.060$, Table 5$)$. An extra $100 \mathrm{kcal} / \mathrm{d}$ energy intake restriction, a unit increase in total fat intake restriction and an additional 10 minutes of exercise would have further reduced BMI by $0.22 \pm 0.08$, $0.10 \pm 0.03$ and $0.24 \pm 0.12 \mathrm{~kg} / \mathrm{m}^{2}$, respectively. Obese adults appeared to respond more to the interventions than normal and overweight adults because a unit increase in BMI at baseline from the mean $\left(30.1 \mathrm{~kg} / \mathrm{m}^{2}\right)$ was associated $(P=0.095)$ with an additional $0.07 \pm 0.04 \mathrm{~kg} / \mathrm{m}^{2} \mathrm{BMI}$ decline. The final mixed-effect model including calorie and fat restrictions, exercise duration, and BMI at baseline

Table 5 Estimates of intervention effect size and total amount of residual heterogeneity $\left(\tau^{2}\right)$ from final mixed-effect models

\begin{tabular}{|c|c|c|c|c|c|c|c|}
\hline \multirow[t]{2}{*}{ Risk factor ${ }^{1}$} & \multicolumn{2}{|c|}{ Overall effect size } & \multicolumn{3}{|c|}{ Effect size change for intervention attributes and other factors } & \multicolumn{2}{|c|}{ Residual heterogeneity } \\
\hline & Estimate \pm SE & $P$-value & Explanatory Variables ${ }^{2}$ & Estimate $\pm \mathrm{SE}^{3}$ & $P$-value & $\tau^{2}$ & $P$-value \\
\hline \multirow[t]{3}{*}{$\mathrm{FI}(\mathrm{mU} / \mathrm{L})$} & $-2.56 \pm 0.58$ & $<0.001$ & Presence of IGT, IR or MetS & $-1.20 \pm 0.69$ & 0.082 & $1.523 \pm 0.773$ & $<0.001$ \\
\hline & & & Calorie restriction (100 kcal/d) & $-0.68 \pm 0.26$ & 0.009 & & \\
\hline & & & Incorporation of resistance training & $1.72 \pm 0.81$ & 0.034 & & \\
\hline \multirow[t]{2}{*}{$\mathrm{FG}(\mathrm{mmol} / \mathrm{L})$} & $-0.18 \pm 0.04$ & $<0.001$ & Presence of IGT, IR or MetS & $-0.08 \pm 0.05$ & 0.098 & $0.010 \pm 0.004$ & $<0.001$ \\
\hline & & & After a follow-up period & $0.13 \pm 0.06$ & 0.034 & & \\
\hline \multirow[t]{3}{*}{$\mathrm{SBP}(\mathrm{mm} \mathrm{Hg})$} & $-2.77 \pm 0.56$ & $<0.001$ & Presence of IGT, IR or MetS & $-3.23 \pm 1.00$ & 0.001 & $2.796 \pm 1.419$ & $<0.001$ \\
\hline & & & Calorie restriction (100 kcal/d) & $-0.64 \pm 0.23$ & 0.006 & & \\
\hline & & & Length of intervention (months) & $0.13 \pm 0.03$ & $<0.001$ & & \\
\hline \multirow[t]{2}{*}{$\mathrm{HDL}(\mathrm{mmol} / \mathrm{L})$} & $-0.015 \pm 0.015$ & 0.305 & Age at baseline (years) & $-0.006 \pm 0.002$ & 0.021 & $0.005 \pm 0.002$ & $<0.001$ \\
\hline & & & Fraction of women participants & $-0.086 \pm 0.046$ & 0.065 & & \\
\hline \multirow[t]{2}{*}{ TAG (mmol/L) } & $-0.258 \pm 0.037$ & $<0.001$ & Calorie restriction (100 kcal/d) & $-0.061 \pm 0.017$ & $<0.001$ & $0.029 \pm 0.009$ & $<0.001$ \\
\hline & & & Baseline TAG (mmol/L) & $-0.243 \pm 0.076$ & 0.001 & & \\
\hline \multirow[t]{4}{*}{$\operatorname{BMI}\left(\mathrm{kg} / \mathrm{m}^{2}\right)$} & $-1.61 \pm 0.13$ & $<0.001$ & Calorie restriction (100 kcal/d) & $-0.22 \pm 0.08$ & 0.003 & $0.353 \pm 0.125$ & $<0.001$ \\
\hline & & & Fat intake restriction (\% Energy) & $-0.10 \pm 0.03$ & 0.003 & & \\
\hline & & & Exercise session duration (10 min) & $-0.24 \pm 0.12$ & 0.055 & & \\
\hline & & & Baseline BMI (kg/m2) & $-0.07 \pm 0.04$ & 0.095 & & \\
\hline
\end{tabular}

${ }^{1}$ Fl: Fasting insulin, FG: Fasting glucose, SBP: Systolic blood pressure, HDL: High density lipoprotein, TAG: Triacylglyceride, BMI: Body mass index.

${ }^{2} I G T=$ Impaired glucose tolerance, $I R=$ Insulin resistance and MetS = Metabolic syndrome.

${ }^{3}$ Standard error of the estimate. 
explained $60 \%$ of the heterogeneity in lifestyle intervention effect on BMI $\left[\tau^{2}=0.881\right.$ (Table 4) vs. 0.353 (Table 5)].

\section{Discussion}

Although meta-analytic applications are increasingly used to summarize results from clinical trials, much uncertainty remains about which approach to use, particularly when significant between-study variability of results or heterogeneity is present [53]. Random-effect methods provide an attractive approach for summarizing heterogeneous results [53]. The lifestyle intervention effects of individual studies included in the present meta-analyses were very heterogeneous (Figures 2, 3 and 4). Therefore, the overall effect sizes and total amount of heterogeneity $\left(\tau^{2}\right)$ was estimated using random-effect approaches. In addition to determining the overall effect size, random-effect approaches allow for exploring factors responsible for heterogeneity. These approaches involve mixed-effect models and are similar to meta-regression approaches. Thus, mixed-effect models were used to explore heterogeneity and estimate the impacts of important attributes of lifestyle interventions on risk factor changes. Such estimates are difficult to find in the literature but would improve diabetes risk prediction models. For example, Appuhamy and colleagues [54] developed a mathematical model for predicting diabetes incidence using BMI. Incorporating the effect size estimates of BMI (Table 5) should enable their model to predict diabetes incidence changes in response to lifestyle modifications. The present metaanalyses summarized effects of lifestyle modifications on other diabetes risk factors such as FI, FG, SBP, HDL and TAG. These factors are also used in mathematical models for predicting diabetes risk [5-7].

Fasting insulin in normoglycemic adults is an important predictor of diabetes risk independent of whether they have insulin resistance or not [55]. Thus, lowering FI could be an option in primary diabetes prevention [55]. The present meta-analyses showed that non-diabetic adults engaging in a diet plus exercise intervention program experienced a $17 \%$ reduction in FI from baseline. Although dietary guidelines in the studies advocate on average a $500 \mathrm{kcal} / \mathrm{d}$ energy restriction, the average net energy intake reduction among intervention participants was $273 \mathrm{kcal} / \mathrm{d}$. A greater compliance with calorie restriction would have further decreased FI because FI was found to decrease linearly by $0.68 \mathrm{mU} / \mathrm{L}$ for each additional $100 \mathrm{kcal} / \mathrm{d}$ reduction. Hence an actual $500 \mathrm{kcal} / \mathrm{d}$ energy restriction would have reduced FI by $4.10 \mathrm{mU} / \mathrm{L}$. Participants with impaired glucose tolerance or insulin resistance had their FI improved to a greater extent than normal participants. When resistance training was incorporated into moderate intensity aerobic exercises, the expected FI improvement for lifestyle interventions was significantly reduced by $1.72 \mathrm{mU} / \mathrm{L}$. This agrees with Holten et al. [56] who demonstrated that strength training could increase FI levels in non-diabetic adults.

Fasting glucose is often used in diabetes risk prediction models [57], although plasma glucose based on the oral glucose tolerance test (OGTT) or the IGT test (IGTT) would better predict diabetes risk [58]. The greater cost and inconvenience associated with these tests generally impede their use in diabetes prediction models [59]. Furthermore, we chose FG over OGTT and IGTT-based glucose because FG was measured in many of the searched studies. Diet and exercise interventions were associated with a decline in FG of $0.18 \mathrm{mmol} / \mathrm{L}$, representing a 3\% drop from baseline $(P<0.001)$. Elevating FG from normoglycemic levels to impaired fasting glucose (IFG) levels doubles the risk of developing diabetes [60,61]. The mean baseline FG of $5.55 \mathrm{mmol} / \mathrm{L}$ indicates that many of the study participants were at a high risk of developing diabetes especially considering the fact that the current cutoff for IFG $(5.60 \mathrm{mmol} / \mathrm{L})$ needs to be reduced for some Western populations [14]. Therefore, this 3\% FG reduction should delay diabetes development in Western adults. Our results showed that continuous dietary counselling and physical activity improvement were necessary for a persistent FG decline. Moreover, the FG levels of adults with abnormalities such as IGT or MetS improved more than those of normal adults.

Hypertension is recognized as an independent predictor of diabetes incidence in various populations. Systolic blood pressure above $120 \mathrm{~mm} \mathrm{Hg}$ is associated with twice the diabetes risk of SBP below $100 \mathrm{~mm} \mathrm{Hg}$ [62]. The present meta-analyses showed that diet plus exercise intervention counseling reduced $(P<0.001)$ SBP by $2.77 \mathrm{~mm} \mathrm{Hg}$. This decline in SBP could be associated with a considerable reduction in diabetes risk as Dotevall et al. [63] reported a 0.10 diabetes hazard ratio increase for every unit increase in SBP above $130 \mathrm{~mm}$ Hg. Normoglycemic adults with abnormalities such as IGT or MetS experienced greater declines in SBP, suggesting more lifestyle intervention benefits for them than for ordinary adults. An improved compliance with calorie restriction guidelines, particularly as the intervention program progressed, could have resulted in a greater SBP decline.

Dyslipidaemia, characterized by elevated TAG and reduced HDL, is usually associated with increased risk of developing diabetes mellitus [64]. Diet plus exercise interventions reduced TAG by $0.258 \mathrm{mmol} / \mathrm{L}(P<0.001)$. This could be associated with a considerably reduced risk of developing diabetes as Tirosh et al. [65] reported a $13 \%$ reduction in diabetes risk for each $0.20 \mathrm{mmol} / \mathrm{L}$ decline in TAG in non-diabetic men. The declines in TAG were greater as baseline TAG levels increased, suggesting more lifestyle intervention benefits for dyslipidaemic adults than normolipidaemic adults. Greater calorie restrictions further enhanced the TAG declines, 
in agreement with the findings of Fontana et al. [66]. When summarized over 30 studies, diet plus exercise interventions did not have a significant impact on HDL. Fourteen studies were associated with reduced HDL (the negative mean differences in Figure 4A) while the rest showed zero or positive responses. Differences in baseline age and percentage of women in the study population explained $29 \%$ of the heterogeneity in HDL responses to lifestyle interventions.

Body mass index is a leading diabetes risk predictor as evidenced by the high correlation between obesity and diabetes prevalence. Curioni and Lourenco [67] and Schaar et al. [68] have summarized diet plus exercise intervention effects on weight loss in adults regardless of diabetes status. These analyses together examined 22 studies and found significant weight loss in response to intervention. We found a consistent reduction in BMI $(P<0.001)$ of $1.61 \mathrm{~kg} / \mathrm{m}^{2}$ (5.3\% from baseline BMI) for diet plus exercise interventions. Such a BMI reduction could be associated with a considerable reduction in diabetes risk as Chiu et al. [69] estimated a 30\% diabetes incidence drop in response to a BMI reduction from 30.0 to $28.0 \mathrm{~kg} /$ $\mathrm{m}^{2}$. Moreover, the mathematical model developed by Appuhamy et al. [53] predicts that a $1.61 \mathrm{~kg} / \mathrm{m}^{2}$ in BMI decline can lead to a $20 \%$ diabetes incidence reduction in non-diabetic middle-aged adults. The heterogeneity in lifestyle intervention effect on BMI was significant (Figure 2B and Table 4). However, degrees of calorie and total fat intake restrictions, duration of exercise and BMI at baseline explained $60 \%$ of the heterogeneity. An additional $100 \mathrm{kcal} / \mathrm{d}$ calorie intake reduction, an extra unit reduction in total fat intake, and extending the exercise session length by 10 minutes could collectively result in an additional $0.56 \mathrm{~kg} / \mathrm{m}^{2}$ decline in BMI. BMI decline significantly improved as baseline BMI increased, suggesting greater lifestyle intervention benefit for obese adults than for adults of normal weight.

Considerable unexplained heterogeneity still remained (residual heterogeneity estimates in Table 5), indicating that factors other than those taken into consideration in the present analyses could be responsible for betweenstudy variability of lifestyle intervention effects. In addition to carbohydrate, fat and protein as explanatory variables, the present meta-analyses also accounted for saturated fat and cholesterol intake restrictions, and fiber intake improvements. However, they did not significantly $(P>0.10)$ explain heterogeneity of lifestyle intervention effects. Data on food sources of fatty acids could have explained heterogeneity to some extent $[70,71]$ but availability of such data was extremely limited in the studies considered. Representations of exercise intensity (e.g., percent maximum heart rate and percent maximum oxygen consumption) may have explained some of the heterogeneity. Furthermore, representation of degree of compliance with diet and exercise guidelines [72] and ethnic composition of participants [73] could have further explained heterogeneity. As the selected articles [20-49] did not consistently report sufficient information to create such variables, we were unable to explore their contribution to heterogeneity.

In summary, lifestyle interventions targeting calorie and total fat intake restrictions and increase in moderate intensity aerobic exercises were associated with significant improvements in diabetes risk factors among normoglycemic adults. Differences in some intervention attributes such as energy and fat intake restrictions, exercise type and session duration, length of intervention, and baseline characteristics of study participants accounted for 23-63\% of the heterogeneity. Having explained such proportions of heterogeneity, lifestyle interventions were associated with significant declines in FI, FG, SBP, TAG, and BMI of $2.56 \mathrm{mU} / \mathrm{L}, 0.18 \mathrm{mmol} / \mathrm{L}, 2.77 \mathrm{~mm} \mathrm{Hg}, 0.258 \mathrm{mmol} / \mathrm{L}$, and $1.61 \mathrm{~kg} / \mathrm{m}^{2}$ respectively in healthy normoglycemic adults. However, normoglycemic adults having abnormalities such as IGT, insulin resistance, metabolic syndrome or hyperlipidemia appeared to benefit more from diet plus exercise intervention programs than healthy normoglycemic adults.

\section{Competing interests \\ The authors declare that they have no competing interests.}

\section{Authors' contributions}

JADRN and JF contributed to conception, design, analysis and interpretation of data and drafted the manuscript. EK and MS contributed to design, analysis and interpretation of data and drafted the manuscript. RY and LPM contributed to conception and drafted the manuscript. All authors read and approved the final manuscript.

\section{Author details}

${ }^{1}$ Department of Animal and Poultry Science, Centre for Nutrition Modelling, University of Guelph, Guelph N1G 2 W1, Ontario, Canada. ²Department of Animal Science, University of California, One Shield Avenue, Davis, CA 95616, USA. ${ }^{3}$ Faculty of Land and Food Systems, University of British Columbia, Vancouver V6T 1Z4, Canada.

Received: 30 May 2014 Accepted: 11 November 2014

Published: 24 November 2014

\section{References}

1. Williams R, Van Gaal L, Lucioni C: Assessing the impact of complications on the costs of type II diabetes. Diabetologia 2002, 45:S13-S17.

2. Zhang P, Zhang X, Brown J, Vistisen D, Sicree R, Shaw J, Nichols G: Global healthcare expenditure on diabetes for 2010 and 2030. Diabetes Res Clin Pract 2010, 87:293-301.

3. Meigs JB: Multiple biomarker prediction of type 2 diabetes. Diabetes Care 2009, 32:1346-1348.

4. Karve A, Hayward RA: Prevalence, diagnosis, and treatment of impaired fasting glucose and impaired glucose tolerance in nondiabetic U.S. adults. Diabetes Care 2010, 33:2355-2359.

5. Hjellvik V, Sakshaug S, Strom H: Body mass index, triglycerides, glucose, and blood pressure as predictors of type 2 diabetes in a middle-aged Norwegian cohort of men and women. Clin Epidemiol 2012, 4:213-224.

6. McNeely MJ, Boyko EJ, Leonetti DL, Kahn SE, Fujimoto WY: Comparison of a clinical model, the oral glucose tolerance test, and fasting glucose for prediction of type 2 diabetes risk in Japanese Americans. Diabetes Care 2003, 26:758-763.

7. Boutayeb A, Chetouani A: A critical review of mathematical models and data used in diabetology. Biomed Eng Online 2006, 5:43. 
8. DerSimonian R, Kacker R: Random-effects models for meta-analysis of clinical trials: an update. Contemp Clin Trials 2007, 28:105-114.

9. Norris SL, Lau J, Smith SJ, Schmid CH, Engelgau MM: Self-management education for adults with type 2 diabetes: a meta-analysis of the effect on glycemic control. Diabetes Care 2002, 25:1159-1171.

10. Brand-Miller J, Hayne S, Petocz P, Colagiuri S: Low-glycemic index diets in the management of diabetes: a meta-analysis of randomized controlled trials. Diabetes Care 2003, 26:2261-2267.

11. Yamaoka K, Tango T: Efficacy of lifestyle education to prevent type 2 diabetes: a meta-analysis of randomized controlled trials. Diabetes Care 2005, 28:2780-2786.

12. Gillies CL, Abrams KR, Lambert PC, Cooper NJ, Sutton AJ, Hsu RT, Khunti K: Pharmacological and lifestyle interventions to prevent or delay type 2 diabetes in people with impaired glucose tolerance: systematic review and meta-analysis. BMJ 2007, 334:299.

13. Norris SL, Zhang X, Avenell A, Gregg E, Schmid CH, Lau J: Long-term non-pharmacological weight loss interventions for adults with prediabetes. Cochrane Database Syst Rev 2005, CD005270.

14. Valdes S, Botas P, Delgado E, Alvarez F, Cadorniga FD: Does the new American Diabetes Association definition for impaired fasting glucose improve its ability to predict type 2 diabetes mellitus in Spanish persons? The Asturias Study. Metabolism 2008, 57:399-403.

15. Wilkins T, Gillies RA, Davies K: EMBASE versus MEDLINE for family medicine searches: can MEDLINE searches find the forest or a tree? Can Fam Physician 2005, 51:848-849.

16. Stefanick ML, Mackey S, Sheehan M, Ellsworth N, Haskell WL, Wood PD: Effects of diet and exercise in men and postmenopausal women with low levels of HDL cholesterol and high levels of LDL cholesterol. N Engl J Med 1998, 339:12-20.

17. Wood PD, Stefanick ML, Williams PT, Haskell WL: The effects on plasma lipoproteins of a prudent weight-reducing diet, with or without exercise, in overweight men and women. N Engl J Med 1991, 325:461-466.

18. Higgins JPT, Altman DG: Chapter 8: assessing risk of bias in included studies. In Cochrane Handbook for Systematic Reviews of Interventions. Edited by Higgins JPT, Green S. Chichester: John Wiley \& Sons Ltd; 2008:187-241.

19. Merrill AL, Watt BK: Energy value of foods: basis and derivation. In Agriculture Handbook. 74th edition. Washington, DC: US Department of Agriculture, Agricultural Research Service; 1973.

20. Straznicky NE, Lambert EA, Grima MT, Eikelis N, Nestel PJ, Dawood T, Schlaich MP, Masuo K, Chopra R, Chopora R, Sari CI, Dixon JB, Tilbrook AJ, Lambert GW: The effects of dietary weight loss with or without exercise training on liver enzymes in obese metabolic syndrome subjects. Diabetes Obes Metab 2012, 14:139-148.

21. Blumenthal JA, Babyak MA, Sherwood A, Craighead L, Lin PH, Johnson J, Watkins LL, Wang JT, Kuhn C, Feinglos M, Hinderliter A: Effects of the dietary approaches to stop hypertension diet alone and in combination with exercise and caloric restriction on insulin sensitivity and lipids. Hypertension 2010, 55:1199-1205.

22. Ibanez J, Izquierdo M, Martinez-Labari C, Ortega F, Grijalba A, Forga L, Idoate F, Garcia-Unciti M, Fernandez-Real JM, Gorostiaga EM: Resistance training improves cardiovascular risk factors in obese women despite a significative decrease in serum adiponectin levels. Obesity (Silver Spring) 2010, 18:535-541.

23. Straznicky NE, Lambert EA, Nestel PJ, McGrane MT, Dawood T, Schlaich MP, Masuo K, Eikelis N, De Courten B, Mariani JA, Esler MD, Socratous F, Chopra R, Sari Cl, Paul E, Lambert GW: Sympathetic neural adaptation to hypocaloric diet with or without exercise training in obese metabolic syndrome subjects. Diabetes 2010, 59:71-79.

24. Roumen C, Corpeleijn E, Feskens EJ, Mensink M, Saris WH, Blaak EE: Impact of 3-year lifestyle intervention on postprandial glucose metabolism: the SLIM study. Diabet Med 2008, 25:597-605.

25. Herder C, Peltonen M, Koenig W, Sutfels K, Lindstrom J, Martin S, Ilanne-Parikka P, Eriksson JG, Aunola S, Keinanen-Kiukaanniemi S, Valle TT, Uusitupa M, Kolb H, Tuomilehto J: Anti-inflammatory effect of lifestyle changes in the Finnish Diabetes Prevention Study. Diabetologia 2009, 52:433-442.

26. Mosca L, Mochari H, Liao M, Christian AH, Edelman DJ, Aggarwal B, Oz MC: A novel family-based intervention trial to improve heart health: FIT Heart: results of a randomized controlled trial. Circ Cardiovasc Qual Outcomes 2008, 1:98-106.

27. Morgan LM, Griffin BA, Millward DJ, DeLooy A, Fox KR, Baic S, Bonham MP, Wallace JM, MacDonald I, Taylor MA, Truby H: Comparison of the effects of four commercially available weight-loss programmes on lipid-based cardiovascular risk factors. Public Health Nutr 2009, 12:799-807.

28. Dale KS, Mann Jl, McAuley KA, Williams SM, Farmer VL: Sustainability of lifestyle changes following an intensive lifestyle intervention in insulin resistant adults: follow-up at 2-years. Asia Pac J Clin Nutr 2009, 18:114-120.

29. Meckling KA, Sherfey R: A randomized trial of a hypocaloric high-protein diet, with and without exercise, on weight loss, fitness, and markers of the Metabolic Syndrome in overweight and obese women. Appl Physiol Nutr Metab 2007, 32:743-752.

30. Burke V, Beilin L, Cutt HE, Mansour J, Williams A, Mori TA: A lifestyle program for treated hypertensives improved health-related behaviors and cardiovascular risk factors, a randomized controlled trial. J Clin Epidemiol 2007, 60:133-141.

31. Bo S, Ciccone G, Baldi C, Benini L, Dusio F, Forastiere G, Lucia C, Nuti C, Durazzo M, Cassader M, Gentile L, Pagano G: Effectiveness of a lifestyle intervention on metabolic syndrome. a randomized controlled trial. J Gen Intern Med 2007, 22:1695-1703.

32. Arciero PJ, Gentile CL, Martin-Pressman R, Ormsbee MJ, Everett M, Zwicky L, Steele CA: Increased dietary protein and combined high intensity aerobic and resistance exercise improves body fat distribution and cardiovascular risk factors. Int J Sport Nutr Exerc Metab 2006, 16:373-392.

33. Brekke HK, Jansson PA, Lenner RA: Long-term (1- and 2-year) effects of lifestyle intervention in type 2 diabetes relatives. Diabetes Res Clin Pract 2005, 70:225-234.

34. Watkins LL, Sherwood A, Feinglos M, Hinderliter A, Babyak M, Gullette E, Waugh R, Blumenthal JA: Effects of exercise and weight loss on cardiac risk factors associated with syndrome X. Arch Intern Med 2003, 163:1889-1895.

35. Lindstrom J, Louheranta A, Mannelin M, Rastas M, Salminen V, Eriksson J, Uusitupa M, Tuomilehto J: The Finnish Diabetes Prevention Study (DPS): lifestyle intervention and 3-year results on diet and physical activity. Diabetes Care 2003, 26:3230-3236.

36. Esposito K, Pontillo A, Di Palo C, Giugliano G, Masella M, Marfella R, Giugliano D: Effect of weight loss and lifestyle changes on vascular inflammatory markers in obese women: a randomized trial. JAMA 2003, 289:1799-1804.

37. Mensink M, Feskens EJ, Saris WH, De Bruin TW, Blaak EE: Study on Lifestyle Intervention and Impaired Glucose Tolerance Maastricht (SLIM): preliminary results after one year. Int J Obes Relat Metab Disord 2003, 27:377-384

38. McAuley KA, Williams SM, Mann Jl, Goulding A, Chisholm A, Wilson N, Story G, McLay RT, Harper MJ, Jones IE: Intensive lifestyle changes are necessary to improve insulin sensitivity: a randomized controlled trial. Diabetes Care 2002, 25:445-452.

39. Miller ER III, Erlinger TP, Young DR, Jehn M, Charleston J, Rhodes D, Wasan SK, Appel $\mathrm{L}$ : Results of the diet, exercise, and weight loss intervention trial (DEW-IT). Hypertension 2002, 40:612-618.

40. Reseland JE, Anderssen SA, Solvoll K, Hjermann I, Urdal P, Holme I, Drevon CA: Effect of long-term changes in diet and exercise on plasma leptin concentrations. Am J Clin Nutr 2001, 73:240-245.

41. Oldroyd JC, Unwin NC, White M, Imrie K, Mathers JC, Alberti KG: Randomised controlled trial evaluating the effectiveness of behavioural interventions to modify cardiovascular risk factors in men and women with impaired glucose tolerance: outcomes at 6 months. Diabetes Res Clin Pract 2001, 52:29-43.

42. Kuller $L H$, Simkin-Silverman $L R$, Wing RR, Meilahn EN, Ives DG: Women's healthy lifestyle project: a randomized clinical trial: results at 54 months. Circulation 2001, 103:32-37.

43. Ornish D, Scherwitz LW, Billings JH, Brown SE, Gould KL, Merritt TA, Sparler S, Armstrong WT, Ports TA, Kirkeeide RL, Hogeboom C, Brand RJ: Intensive lifestyle changes for reversal of coronary heart disease. JAMA 1998, 280:2001-2007.

44. Wing RR, Venditti E, Jakicic JM, Polley BA, Lang W: Lifestyle intervention in overweight individuals with a family history of diabetes. Diabetes Care 1998, 21:350-359.

45. Simkin-Silverman L, Wing RR, Hansen DH, Klem ML, Pasagian-Macaulay AP, Meilahn EN, Kuller LH: Prevention of cardiovascular risk factor elevations in healthy premenopausal women. Prev Med 1995, 24:509-517.

46. Hellenius ML, de Faire U, Berglund B, Hamsten A, Krakau I: Diet and exercise are equally effective in reducing risk for cardiovascular disease. results of a randomized controlled study in men with slightly to moderately raised cardiovascular risk factors. Atherosclerosis 1993, 103:81-91. 
47. Svendsen OL, Hassager C, Christiansen C: Effect of an energy-restrictive diet, with or without exercise, on lean tissue mass, resting metabolic rate, cardiovascular risk factors, and bone in overweight postmenopausal women. Am J Med 1993, 95:131-140.

48. Page RC, Harnden KE, Walravens NK, Onslow C, Sutton P, Levy JC, Hockaday DT, Turner RC: 'Healthy living' and sulphonylurea therapy have different effects on glucose tolerance and risk factors for vascular disease in subjects with impaired glucose tolerance. Q J Med 1993, 86:145-154.

49. Schuler G, Hambrecht R, Schlierf G, Niebauer J, Hauer K, Neumann J, Hoberg E, Drinkmann A, Bacher F, Grunze M, Kubler W: Regular physical exercise and low-fat diet. effects on progression of coronary artery disease. Circulation 1992, 86:1-11.

50. Viechtbauer W: Conducting meta-analyses in $\mathrm{R}$ with the metafor package. J Stat Softw 2010, 36:1-48.

51. Sutton AJ, Duval SJ, Tweedie RL, Abrams KR, Jones DR: Empirical assessment of effect of publication bias on meta-analyses. BMJ 2000, 320:1574-1577.

52. Viechtbauer W, Cheung MWL: Outlier and influence diagnostics for meta-analysis. Res Synthesis Methods 2010, 1:112-125.

53. Engels EA, Schmid CH, Terrin N, Olkin I, Lau J: Heterogeneity and statistical significance in meta-analysis: an empirical study of 125 meta-analyses. Stat Med 2000, 19:1707-1728.

54. Appuhamy JA, Kebreab E, France J: A mathematical model for determining age-specific diabetes incidence and prevalence using body mass index. Ann Epidemiol 2013, 23:248-254.

55. Lillioja S, Mott DM, Spraul M, Ferraro R, Foley JE, Ravussin E, Knowler WC, Bennett PH, Bogardus C: Insulin resistance and insulin secretory dysfunction as precursors of non-insulin-dependent diabetes mellitus. prospective studies of Pima Indians. N Engl J Med 1993, 329:1988-1992.

56. Holten MK, Zacho M, Gaster M, Juel C, Wojtaszewski JF, Dela F: Strength training increases insulin-mediated glucose uptake, glut4 content, and insulin signaling in skeletal muscle in patients with type 2 diabetes. Diabetes 2004, 53:294-305.

57. Abdul-Ghani MA, Williams K, DeFronzo RA, Stern M: What is the best predictor of future type 2 diabetes? Diabetes Care 2007, 30:1544-1548.

58. Stern MP, Williams K, Haffner SM: Identification of persons at high risk for type 2 diabetes mellitus: do we need the oral glucose tolerance test? Ann Intern Med 2002, 136:575-581.

59. Wang JJ, Yuan SY, Zhu LX, Fu HJ, Li HB, Hu G, Tuomilehto J: Effects of impaired fasting glucose and impaired glucose tolerance on predicting incident type 2 diabetes in a Chinese population with high post-prandial glucose. Diabetes Res Clin Pract 2004, 66:183-191.

60. Rathmann W, Strassburger K, Heier M, Holle R, Thorand B, Giani G, Meisinger C: Incidence of type 2 diabetes in the elderly German population and the effect of clinical and lifestyle risk factors: KORA S4/F4 cohort study. Diabet Med 2009, 26:1212-1219.

61. Janghorbani M, Amini M: Comparison of systolic and diastolic blood pressure with pulse pressure and mean arterial pressure for prediction of type 2 diabetes: the Isfahan Diabetes Prevention Study. Endokrynol Pol 2011, 62:324-330.

62. Geiss LS, Pan L, Cadwell B, Gregg EW, Benjamin SM, Engelgau MM: Changes in incidence of diabetes in U.S. adults, 1997-2003. Am J Prev Med 2006, 30:371-377.

63. Dotevall A, Johansson S, Wilhelmsen L, Rosengren A: Increased levels of triglycerides, BMI and blood pressure and low physical activity increase the risk of diabetes in Swedish women. a prospective 18-year follow-up of the BEDA study. Diabet Med 2004, 21:615-622.

64. Fukui M, Tanaka M, Toda H, Senmaru T, Sakabe K, Ushigome E, Asano M, Yamazaki M, Hasegawa G, Imai S, Nakamura N: Risk factors for development of diabetes mellitus, hypertension and dyslipidemia. Diabetes Res Clin Pract 2011, 94:e15-e18.

65. Tirosh A, Shai I, Bitzur R, Kochba I, Tekes-Manova D, Israeli E, Shochat T, Rudich $A$ : Changes in triglyceride levels over time and risk of type 2 diabetes in young men. Diabetes Care 2008, 31:2032-2037.

66. Fontana L, Meyer TE, Klein S, Holloszy JO: Long-term calorie restriction is highly effective in reducing the risk for atherosclerosis in humans. Proc Natl Acad Sci U S A 2004, 101:6659-6663.

67. Curioni CC, Lourenco PM: Long-term weight loss after diet and exercise: a systematic review. Int J Obes (Lond) 2005, 29:1168-1174.
68. Schaar B, Moose-Thiele C, Platen P: Effects of exercise, diet, and a combination of exercise and diet in overweight and obese adults - a meta-analysis of the data. Open Sport Med J 2010, 4:17-28.

69. Chiu M, Austin PC, Manuel DG, Shah BR, Tu JV: Deriving ethnic-specific BM cutoff points for assessing diabetes risk. Diabetes Care 2011, 34:1741-1748.

70. Mozaffarian D, Cao H, King IB, Lemaitre RN, Song X, Siscovick DS, Hotamisligil GS: Trans-palmitoleic acid, metabolic risk factors, and new-onset diabetes in U.S. adults: a cohort study. Ann Intern Med 2010, 153:790-799.

71. Micha R, Mozaffarian D: Saturated fat and cardiometabolic risk factors, coronary heart disease, stroke, and diabetes: a fresh look at the evidence. Lipids 2010, 45:893-905.

72. Rickel KA, Milsom VA, Ross KM, Hoover VJ, Peterson ND, Perri MG: Differential response of African American and Caucasian women to extended-care programs for obesity management. Ethn Dis 2011, 21:170-175.

73. Hertogh EM, Schuit AJ, Peeters PH, Monninkhof EM: Noncompliance in lifestyle intervention studies: the instrumental variable method provides insight into the bias. J Clin Epidemiol 2010, 63:900-906.

doi:10.1186/1758-5996-6-127

Cite this article as: Appuhamy et al:: Effects of diet and exercise interventions on diabetes risk factors in adults without diabetes: meta-analyses of controlled trials. Diabetology \& Metabolic Syndrome 2014 6:127.

\section{Submit your next manuscript to BioMed Central and take full advantage of:}

- Convenient online submission

- Thorough peer review

- No space constraints or color figure charges

- Immediate publication on acceptance

- Inclusion in PubMed, CAS, Scopus and Google Scholar

- Research which is freely available for redistribution 